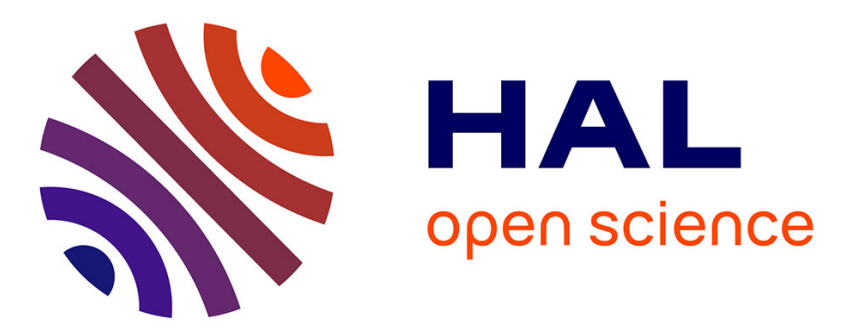

\title{
A new species of Psilogamasus Athias-Henriot, 1969 from China and redefinition of the genus (Parasitiformes: Parasitidae)
}

\author{
Mao-Yuan Yao, Tian-Ci Yi, Dao-Chao Jin, Jian-Jun Guo
}

\section{- To cite this version:}

Mao-Yuan Yao, Tian-Ci Yi, Dao-Chao Jin, Jian-Jun Guo. A new species of Psilogamasus AthiasHenriot, 1969 from China and redefinition of the genus (Parasitiformes: Parasitidae). Acarologia, 2020, 60 (4), pp.831-841. 10.24349/acarologia/20204404 . hal-03010274

\section{HAL Id: hal-03010274 \\ https://hal.science/hal-03010274}

Submitted on 17 Nov 2020

HAL is a multi-disciplinary open access archive for the deposit and dissemination of scientific research documents, whether they are published or not. The documents may come from teaching and research institutions in France or abroad, or from public or private research centers.
L'archive ouverte pluridisciplinaire HAL, est destinée au dépôt et à la diffusion de documents scientifiques de niveau recherche, publiés ou non, émanant des établissements d'enseignement et de recherche français ou étrangers, des laboratoires publics ou privés.

\section{(c)(1)}

Distributed under a Creative Commons Attribution| 4.0 International License 


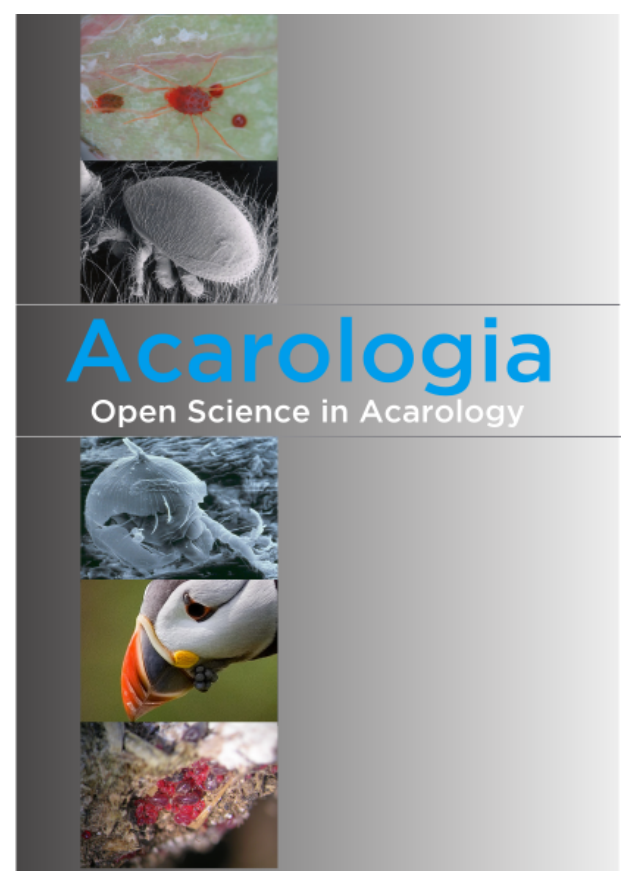

\section{Acarologia}

A quarterly journal of acarology, since 1959

Publishing on all aspects of the Acari

All information:

http://www1.montpellier.inra.fr/CBGP/acarologia/ acarologia-contact@supagro.fr

\section{OPEN ACCESS}

\section{Acarologia is proudly non-profit, with no page charges and free open access}

Please help us maintain this system by encouraging your institutes to subscribe to the print version of the journal and by sending us your high quality research on the Acari.

Subscriptions: Year 2020 (Volume 60): $450 €$ http://www1.montpellier.inra.fr/CBGP/acarologia/subscribe.php

Previous volumes (2010-2018): $250 €$ / year (4 issues)

Acarologia, CBGP, CS 30016, 34988 MONTFERRIER-sur-LEZ Cedex, France

ISSN 0044-586X (print), ISSN 2107-7207 (electronic)

The digitalization of Acarologia papers prior to 2000 was supported by Agropolis Fondation under the reference ID 1500-024 through the « Investissements d'avenir » programme

(Labex Agro: ANR-10-LABX-0001-01)
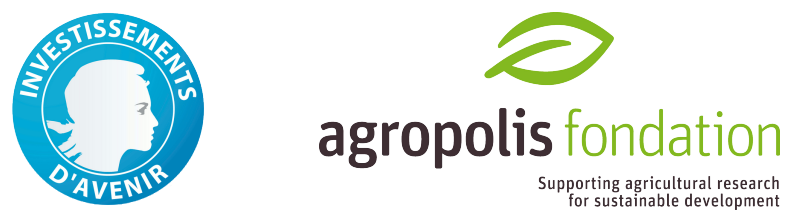

Acarologia is under free license and distributed under the terms of the

Creative Commons-BY-NC-ND which permits unrestricted non-commercial use, distribution, and reproduction in any medium, provided the original author and source are credited. 


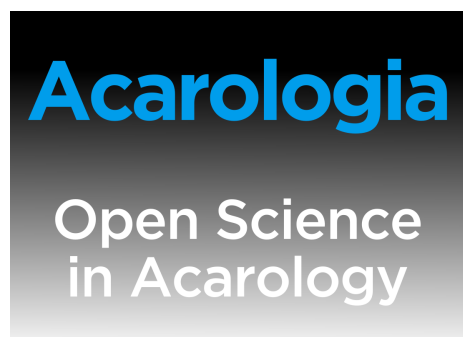

A new species of Psilogamasus Athias-Henriot, 1969 from China and redefinition of the genus (Parasitiformes: Parasitidae)

\author{
Mao-Yuan Yao ${ }^{a, b}$, Tian-Ci Yi ${ }^{a, b}$, Dao-Chao Jin ${ }^{a, b}$, Jian-Jun Guo $^{a, b}$ \\ ${ }^{a}$ Institute of Entomology, Guizhou University, Guiyang, Guizhou,550025, P.R. China. \\ ${ }^{b}$ Guizhou Provincial Key Laboratory for Agricultural Pest Management of the Mountainous Region, \\ Scientific Observing and Experimental Station of Crop Pest in Guiyang, Ministry of Agriculture, P. R. \\ China, Guiyang 550025. P. R. China.
}

\section{Orginal research}

\section{ABSTRACT}

A new species, Psilogamasus pentatideus Yao \& Jin n. sp., is described based on adults from Hainan province, China. Taiwanoparasitus Tseng is newly synonymized with Psilogamasus Athias-Henriot. Four species, Taiwanoparasitus pentasetosus Tseng, 1995, Vulgarogamasus brachysternalis Ma \& Lin, 2005, V. longascidiformis Ma \& Lin, 2005, V. lingulatus Bai \& Ma, 2013, are transferred to Psilogamasus as new combinations. A key to the known species of the genus is given and the diagnosis of the genus is revised.

Keywords Psilogamasus; Taiwanoparasitus; synonyms; genus redefinition; new combination; identification key

Zoobank http://zoobank.org/439E834B-E519-4E52-B18B-5E4930A2E495

\section{Introduction}

Mites of the family Parasitidae Oudemans, 1901 are distributed worldwide and occur in animal dung, compost, leaf litter, moss, rotting seaweed, soil and the nests of small mammals and birds (Micherdziński 1969; Hyatt 1980; Halliday et al. 2005; Witaliński 2017). The family comprises 46 genera in two subfamilies (Hrúzová \& Fend'a 2018; Juvara-Bals 2019). Bibliographic analysis by the authors shows that about 130 species in 15 genera of Parasitidae have been described from China.

The monotypic genus Psilogamasus, belonging to the subfamily Parasitinae, was erected by Athias-Henriot (1969) on the basis of Psilogamasus hurlbutti Athias-Henriot, 1969 collected in Tanzania. The characters regarded as diagnostic by Athias-Henriot (1969) were: podonotum with 18 pairs of setae, of which seven pairs are short, opisthonotum with six pairs of setae, opisthogaster with five pairs of setae, peritrematal shields free posteriorly, endogynium large, sac-shaped, movable digit of female chelicera with four teeth, telotarsus IV with a trichocystic seta (Athias-Henriot, 1969). This species has since been recorded in North Carolina (Hennesey \& Farrier 1989), so may be more widely distributed through the world.

In this regard, Taiwanoparasitus Tseng, 1995 is very similar to Psilogamasus. It was a monotypic genus when erected, with Taiwanoparasitus pentasetosus Tseng, 1995 as type species. Recently, Hrúzová \& Fend'a (2018) transferred two species from the Chinese mainland, Vulgarogamasus brachysternalis Ma \& Lin, 2005 and V. longascidiformis Ma \& Lin, 2005, to Taiwanoparasitus (Tseng 1995; Ma \& Lin 2005). The generic diagnosis of Taiwanoparasitus was derived from only females of the type species $T$. pentasetosus and was proposed on the basis of its combination of five pairs of opisthonotal setae and stigmatal position. Hrúzová 
\& Fend'a (2018) pointed out that this genus has similarity with Psilogamasus Athias-Henriot, 1969, which Tseng (1995) did not compare when proposing Taiwanoparasitus, and these genera need further work to determine their taxonomic status.

Herein, we describe a new species of Psilogamasus, move four species into the genus, and redefine the genus. This broader concept of the genus necessitates the synonymy of Taiwanoparasitus under Psilogamasus, as alluded to by Hrúzová \& Fend'a (2018).

\section{Material and methods}

Mites were extracted from rotten leaves by Berlese Tullgren funnels, each equipped with a 40 or 60 Watt bulb, and stored in $75 \%$ alcohol. Mites were cleared in Nesbitt's solution and then mounted on slides in Hoyer's medium. Specimens were examined using a Nikon DS-Ri2 microscope. All measurements were taken with the software Leica Application Suite V 4.4 for all available specimens and are given in micrometers $(\mu \mathrm{m})$.

Idiosomal length was measured, in dorsal view, from the anterior margin of the podonotal shield to the posterior margin of the idiosoma and idiosomal width was taken at the widest point. The length of dorsal shields was measured medially from the anterior to the posterior margins, the width was measured at their widest points. Legs were measured from the base of the coxa to the distal end of the tarsus, excluding the ambulacrum (stalk, claws and pulvillus), and the palps were measured from the base of the trochanter to the distal end of the tarsus. Setae were measured from the alveolus to the apex of the seta.

The system of idiosomal setal nomenclature follows Hyatt (1980). Terminology for the leg chaetotaxy follows Evans (1963a), palp chaetotaxy follows Evans (1963b) and adenotaxy and poroidotaxy follows Athias-Henriot $(1971,1975)$ as adapted Yao et al. $(2019,2020)$.

\section{Redefinition of Psillogamasus with four new combinations}

\section{Genus Psilogamasus Athias-Henriot, 1969}

Type species: Psilogamasus hurlbutti Athias-Henriot, 1969

\section{Diagnosis}

Both sexes. Dorsal idiosoma with less than 30 pairs of setae, of which seven or eight pairs of podonotal setae very short; setae $z 5$ of dorsal hexagon similar to $j 5$ and $j 6$ in form (smooth), while different in length ( $z 5$ longer); presternal platelets absent; tritosternum biramous; opisthogaster bearing five to six pairs of setae; setae $Z V 1$ and gland pores gv2 absent; seta al of palp femur comblike, all and al2 of palp genu entire and spatulate distally; corniculi small; epistome trispinate, central prong apically with two to five branches or multidentate, lateral prongs spiculate with bifid apex or two to four apical branches.

Female with separated podonotal shield that with 16-18 pairs of setae, and opisthonotal shield that with five to six pairs of setae; genital shield triangular or subtriangular; peritrematal shield anteriorly fused to podonotal shield and posteriorly free; movable digit of chelicerae with three or four teeth; setae $a v 1$ and $a v 2$ on femur II, $a v 1$ on genu II and tibia II acicular.

Male with holodorsal shield and transverse suture in central region; movable digit of chelicera with five or six teeth; two ventral setae on femur II modified into spurs fused at base; one ventral seta on genu II and tibia II each modified into spur.

\section{Remarks}

In Hrúzová \& Fend'a (2018), the genera Psilogamasus and Taiwanoparasitus were separated by the number of setae on the opisthonotal shield (six and five pairs, respectively) and the presence of trichocystic seta $p d 2$ on telotarsus IV (present and absent, respectively). However, 
Table 1 Morphological variations in females of Psilogamasus brachysternalis (Ma \& Lin, 2005) n. comb., Psilogamasus hurlbutti (AthiasHenriot, 1969), Psilogamasus lingulatus (Bai \& Ma, 2013) n. comb., Psilogamasus longascidiformis (Ma \& Lin, 2005) n. comb., Psilogamasus pentasetosus (Tseng, 1995) n. comb. and Psilogamasus pentatideus Yao \& Jin n. sp..

\begin{tabular}{|c|c|c|c|c|c|c|c|}
\hline Mite & $\begin{array}{l}\text { Setal number on } \\
\text { podonotal shield }\end{array}$ & $\begin{array}{l}\text { Setal number on } \\
\text { opisthonotal } \\
\text { shield }\end{array}$ & $\begin{array}{l}\text { Setal number on } \\
\text { opisthogastric } \\
\text { shield }\end{array}$ & $\begin{array}{l}\text { Dental numbers of } \\
\text { movable digit on } \\
\text { chelicera }\end{array}$ & $\begin{array}{l}\text { Dental numbers } \\
\text { of fixed digit on } \\
\text { chelicera }\end{array}$ & Peritrematal groove & $\begin{array}{l}\text { Shape of } \\
\text { setae } j 1\end{array}$ \\
\hline P. brachysternalis & 18 pairs & 5 pairs & 5 pairs & 3 & 5 & $\begin{array}{l}\text { extending anteriorly and close to } \\
\text { posterior end of coxa III }\end{array}$ & smooth \\
\hline P. hurlbutti & 18 pairs & 6 pairs & 5 pairs & 4 & 7 & $\begin{array}{l}\text { not mentioned in the regional } \\
\text { description }\end{array}$ & smooth \\
\hline$P$. lingulatus & 16-18 pairs & 6 pairs & 5 pairs & 4 & 5 & $\begin{array}{l}\text { extending anteriorly and beyond } \\
\text { coxa II }\end{array}$ & $\begin{array}{l}\text { slightly } \\
\text { pilose }\end{array}$ \\
\hline P. longascidiformis & 17 pairs & 5 pairs & 5 pairs & 3 & 5 & $\begin{array}{l}\text { extending anteriorly and close to } \\
\text { posterior end of coxa III }\end{array}$ & smooth \\
\hline P. pentasetosus & 16 pairs & 5 pairs & 6 pairs & 4 & 6 & $\begin{array}{l}\text { extending anteriorly and beyond } \\
\text { coxa II }\end{array}$ & smooth \\
\hline $\begin{array}{l}\text { P. pentatideus } \\
\text { Yao \& Jin n. sp. }\end{array}$ & 18 pairs & 5 pairs & 5 pairs & 4 & 7 & $\begin{array}{l}\text { extending anteriorly and beyond } \\
\text { coxa II }\end{array}$ & smooth \\
\hline
\end{tabular}

they also discussed the lack of data on the latter character, leaving the number of opisthosomal shield setae as the critical feature for defining the genera.

In regards to the number of opisthosomal shield setae, we explored the constituent species of both genera and found no other character states that were linked with this feature (Table 1). Therefore, we concluded this was best considered an intrageneric feature, as suspected by Hrúzová \& Fend’a (2018). Therefore, we consider Taiwanoparasitus a junior synonym of Psilogamasus.

The above action of synonymizing Taiwanoparasitus under Psilogamasus leads to several new combinations, i.e., T. brachysternalis, $T$. longascidiformis and T. pentasetosus are now included in the genus Psilogamasus.

Psilogamasus brachysternalis (Ma \& Lin, 2005) n. comb.

Original designation and recent revision: Vulgarogamasus brachysternalis Ma \& Lin, 2005: p. 73-74; Taiwanoparasitus brachysternalis (Ma \& Lin) Hrúzová \& Fend’a, 2018: p. 39

Psilogamasus longascidiformis (Ma \& Lin, 2005) n. comb.

Original designation and recent revision: Vulgarogamasus longascidiformis Ma \& Lin, 2005: p. 74-75; Taiwanoparasitus longascidiformis (Ma \& Lin) Hrúzová \& Fend’a, 2018: p. 39 .

Psilogamasus pentasetosus (Tseng, 1995) n. comb.

Original designation: Taiwanoparasitus pentasetosus Tseng, 1995: p. 34-35

Based on the previous records of Athias-Henriot (1969), Tseng (1995), Ma \& Lin (2005), Bai \& Ma (2013), and newly found collections in this study, the geographical distribution of Psilogamasus is limited to Tanzania and China, with the possible adventive presence of $P$. hurlbutti in North America (Hennesey \& Farrier 1989).

We conclude that the species Vulgarogamasus lingulatus Bai \& Ma, 2013 may also be moved into the genus Psilogamasus.

Psilogamasus lingulatus (Bai \& Ma, 2013) n. comb.

Original designation: Vulgarogamasus lingulatus Bai \& Ma, 2013: p. 71-73

The genus Vulgarogamasus, established by Tichomirov (1969), markedly differs from Psilogamasus by having setae $z 5$ of the dorsal hexagon very similar to $j 5$ and $j 6$ in length, and both podonotal and opisthonotal shields with more than 20 pairs of setae. The female of $V$. lingulatus differs from the other species of Vulgarogamasus by the following characteristics: $z 5$ longer than $j 5$ and $j 6$, opisthonotal shield with only 6 pairs of setae, peritrematal shield free posteriorly and central prong of epistome multidentate distally and lateral ones spiculate or 
bifid apically. The features of female $V$. lingulatus conform with Psilogamasus: peritrematal shield anteriorly fused to podonotal shield, posteriorly free; 16-18 pairs of acicular setae on the podonotal shield, of which 8 pairs are tiny; six pairs of acicular setae on opisthonotal shield; opisthogaster bearing 5 pairs of setae, setae $Z V 1$ and gland pores gv2 absent; central prong of epistome multidentate distally and lateral ones spiculate or bifid apically; endogynial sac large.

The description of $P$. pentatideus n. sp. and the additions of $P$. brachysternalis n. comb., $P$. longascidiformis n. comb. and $P$. lingulatus n. comb., $P$. pentasetosus n. comb. permit a revision of the genus diagnosis. Within this genus, the number of setae on the idiosomal shields, dental numbers of cheliceral digits, and the length of the peritrematal groove are variable (Table 1).

However, we add further character states to define the female of Psilogamasus. On the venter, setae $Z V 1$ is absent (erroneously named $Z V 1$ by Tseng (1995) was $Z V 2$ ) and peritrematal shield free posteriorly; on the dorsum the total number of dorsal setae is less than 30 pairs, and of which $z 5, j 5, j 6$ are smooth; and setae $a v 1$ and $a v 2$ on femur II, $a v 1$ on genu II and tibia II are acicular.

The diagnostic definition of Psilogamasus male, with the most conspicuous features, can also now be proposed as: the holodorsal shield bears less than 30 pair of setae; the movable digit of the chelicera has five or six teeth, which is rare in Parasitidae; setae $a v 1$ and $a v 2$ on femur II, $a v 1$ on genu II and tibia II are spur-like.

\section{Psilogamasus pentatideus Yao \& Jin n. sp. (Figures 1-4)}

Zoobank: 8EC705F3-8236-420F-86E2-FBABCA5BD68D

\section{Material examined}

Holotype, female (slide no. HN 201801160801), encountered in rotten leaves, Wuzhi Mountain $\left(109^{\circ} 40^{\prime} 5^{\prime \prime} \mathrm{E}, 18^{\circ} 51^{\prime} 13^{\prime \prime} \mathrm{N}\right.$, ca. $638 \mathrm{~m}$ a.s.l. above sea level), Hainan Province, China, Jan. 2018. Paratypes, seven females (HN 20180160802-HN 201801160807) and four males (HN 201801160808-HN 201801160812) same collection data as the holotype. The holotype and all other specimens are deposited in the Institute of Entomology, Guizhou University, Guiyang, China (GUGC).

\section{Description}

\section{Female}

$(\mathrm{n}=8$; Figures $1-2)$

Dorsum (Figure 1A) - Idiosoma weakly sclerotized, length 679-704, width 481-498, oval in outline. Podonotal and opisthonotal shields separated and with rough reticulation. Podonotal region with 19 pairs of setae, of which 18 pairs on podonotal shield (length 341-358, width 387-395) and a pair (seta $r 6$ ) off shield. Seven pairs of pore-like structures on podonotal shield visible. Opisthonotal region bearing seven pairs of setae, of which $J 1-J 3$ and $Z 1-Z 2$ on opisthonotal shield (length 254-270, width 331-342), J4 and Z3 off shield. Two pairs of pore-like structures on opisthonotal shield visible. All dorsal setae smooth. Seta $r 3$ (163-167) longer and stouter than others, setae $j 2, j 3, z 2, s 3, r 2, r 4, r 6, Z 2$ and $Z 3$ tiny, equal in length (8-10). Lengths of other dorsal setae on shields: j1 74-78, j4 68-71, j5 68-72, j6 96-98, z1 25-27, z4 61-63, z5 124-128, z6 77-80, s4 68-71, s5 68-70, s6 19-21, J1 105-109, J2 110-114, J3 107-111, J4 86-89, Z1 76-81.

Venter (Figures 1B-1C) - Tritosternum (Figure 1C) with two pilose laciniae (95-109) and a smooth base (48-53). Presternal platelets absent. Sternal shield reticulated, fused to endopodals between coxae I-II and II-III, bearing three pairs of setae (st1-3), st1 48-52, st2 and st3 36-39 in length, and two pairs of poroids (iv1-2). Anterior as well as posterior margin of sternal shield with an irregular concavity medially. Paragynial shields separated from sternal shield by medially arched groove, bearing setae st4 (38-40) and poroids $i v 3$. Epigynial shield 


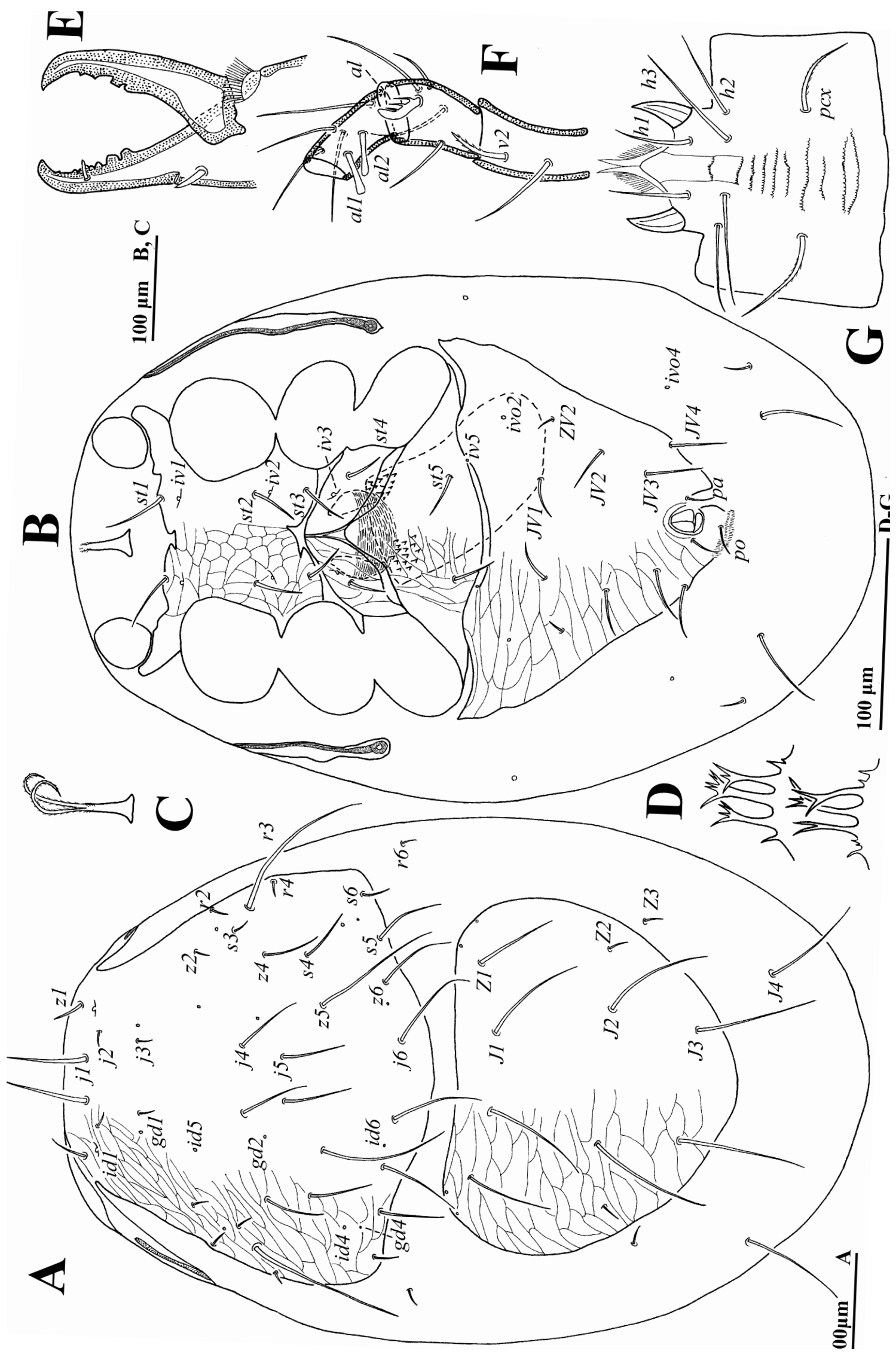

Figure 1 Psilogamasus pentatideus Yao \& Jin n. sp., female. A - Dorsum; B - Venter; C - Tritosternum; D - Epistome; E - Chelicera; F. Palpus; G. Subcapitulum. 


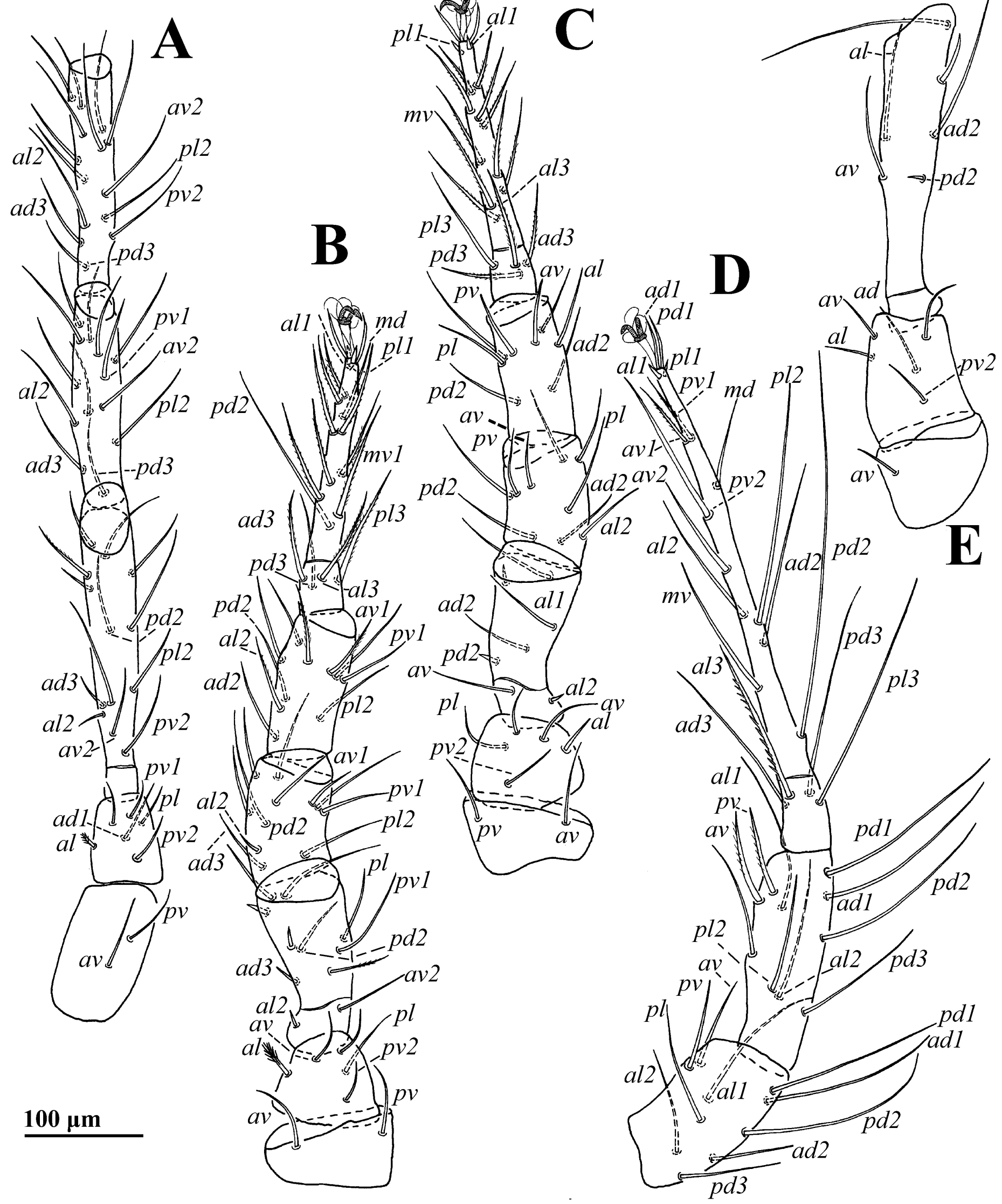

Figure 2 Psilogamasus pentatideus Yao \& Jin n. sp., female. A - Coxa-tibia of leg I; B - Leg II; C - Leg III; D - Genu-tarsus of leg IV; E Coxa-femur of leg IV. 
separated from opisthogastric shield by a transverse suture, bearing setae st5 (39-42) and poroids $i v 5$. Anterior margin of epigynial shield with smooth rounded apex. Endogynium tongue-shaped with some small teeth on each side of the leading edge, and anterolaterally with two inner reniform structures underneath paragynial shields, connected by a ribbon structure. Setae $Z V 1$ and gland pores $g v 2$ absent. Opisthogastric shield reticulate, bearings five pairs of setae (JV1-4 and ZV2). Setae pa and po equal in length (18-21). Peritrematal groove length 209-230, extending to anterior level of coxa II. Peritrematal shield fused with podonotal shield anteriorly and separated at level of seta $j 2$. Opisthogastric soft cuticle with two pairs of setae and two pairs of pore-like structures. All ventral setae smooth. Lengths of setae on opisthogastric shield: JV1 34-36, JV2 39-42, JV3 39-42, JV4 54-56, ZV2 12-13.

Gnathosoma (Figures 1D-1G) - Epistome (Figure 1D) with three prongs, divided into two to five branches apically, emerging from base with one to three small teeth on each side. Movable digit of chelicera with four teeth, proximal two teeth robust and larger than two distal ones, and with arthrodial brush at base, fixed digit with seven teeth, distal one smaller and more pointed than others, an acicular pilus dentilis and one smooth dorsal seta near base (Figure 1E). Palp length 241-253; trochanter bearing two pairs of setae, $v 2$ pilose; femur with five setae, of which al comblike; genu with six pairs of setae, of which all and al2 spatulate distally, remaining setae smooth. Trochanter, femur and genu of palp as in Figure 1F. Corniculus length 27-34, short and horn-shaped; internal mala acute with plate-like bases bearing numerous pili; deutosternal groove with ten denticulate rows. Setae $h 1-h 3$ smooth, $h 140-43, h 241-44, h 3$ 57-61 in length; $p c x$ slightly pilose, 54-57 in length (Figure $1 \mathrm{G}$ ).

Legs (Figure 2) — Lengths of legs I-IV: 990-1003, 639-664, 665-679 and 1017-1129. Leg IV longer than other legs. Most leg setae smooth and setae on femur, genu and tibia of leg IV usual longer than other. Setae $a l$ on trochanter I-II stout and pilose. Most setae on tarsi II-III slightly pinnate. Setae all and pll on tarsi II-IV spur-like. Setae al3 stout and pectinate and setae $p d 2$ on tarsus IV, length 332-339, longer than all other setae on tarsus IV. Chaetotactic formulae of leg I-IV as follows: coxa $(0$ 0/1, 0/1 0), (0 0/1, 0/1 0), (0 0/1, 0/1 0), (0 0/1, 0/0 0); trochanter $(11 / 1,0 / 21),(10 / 1,0 / 21),((11 / 1,0 / 20),(11 / 1,0 / 20)$; femur $(23 / 2,2 / 22),(2$ $3 / 2,2 / 1$ 1), (1 2/1, 2/0 0), (1 2/1, 2/0 0); genu (2 3/2,3/1 2), (2 3/1, 2/1 2), (1 2/1, 2/1 2), (2 2/1, $3 / 1$ 1); tibia $(23 / 2,3 / 22),(2$ 2/1, 2/1 2), (1 2/1, 2/1 1), (2 1/1,3/1 2); and tarsi II-IV $(33 / 2,1 / 1$, $3 / 23)$.

\section{Male}

$(\mathrm{n}=4$; Figures 3-4)

Dorsum (Figure 3A) - Idiosoma length 510-532, width 341-347. Reticulate holodorsal shield covering entire dorsum, with a suture closely anterior to setae $J 1$, not reaching lateral margin of idiosoma. Podonotal region with 19 pairs of setae. Opisthonotal region bearing seven pairs of setae. All setae smooth and inserted on reticulate holodorsal shield. Setae $j 2, j 3$, $z 2, s 3, r 2, r 4, r 6$ similar in length (8-10), finer and shorter than others. Lengths of other dorsal setae: $j 1$ 63-64, j4 56-58, j5 67-69, j6 79-82, z1 22-23, z4 61-63, z5 112-116, z6 66-67, r3 141-145, s4 52-54, s5 59-61, s6 19-21, J1 89-93, J2 83-85, J3 90-94, J4 71-73, Z1 57-58, Z2 14-15, 14-15.

Venter (Figure 3B) - Tritosternum with two pilose laciniae (108-114), and a smooth base (28-32). Presternal shields absent. Genital lamina poorly visible, anteriorly extending to form a hyaline and smooth protrusion. Sternogenital shield reticulated, bearing five pairs of setae (st1-5), subequal in length (39-43), and three pairs of poroids. Anterior margin of sternogenital shield concave, posterior margin separated from opisthogastric region by a transverse suture. Opisthogastric region reticulated and with seven pairs of smooth setae, of which setae $Z V 2$ and one pair of lateral setae short and fine, equal in length (8-10); setae $J V 1$ 31-32, JV2 30-32, JV3 40-42, JV4 40-42, JV5 61-64 in length. Setae $p a$ and po equal in length (17-19). Peritrematal groove as in female, length 191-224. Peritrematal shield fused to opisthogastric shield.

Gnathosoma (Figures 4A-4D) - Epistome (Figure 4A) with three prongs, split into two to three branches distally, from base with one to three small teeth on each side. Subcapitulum 


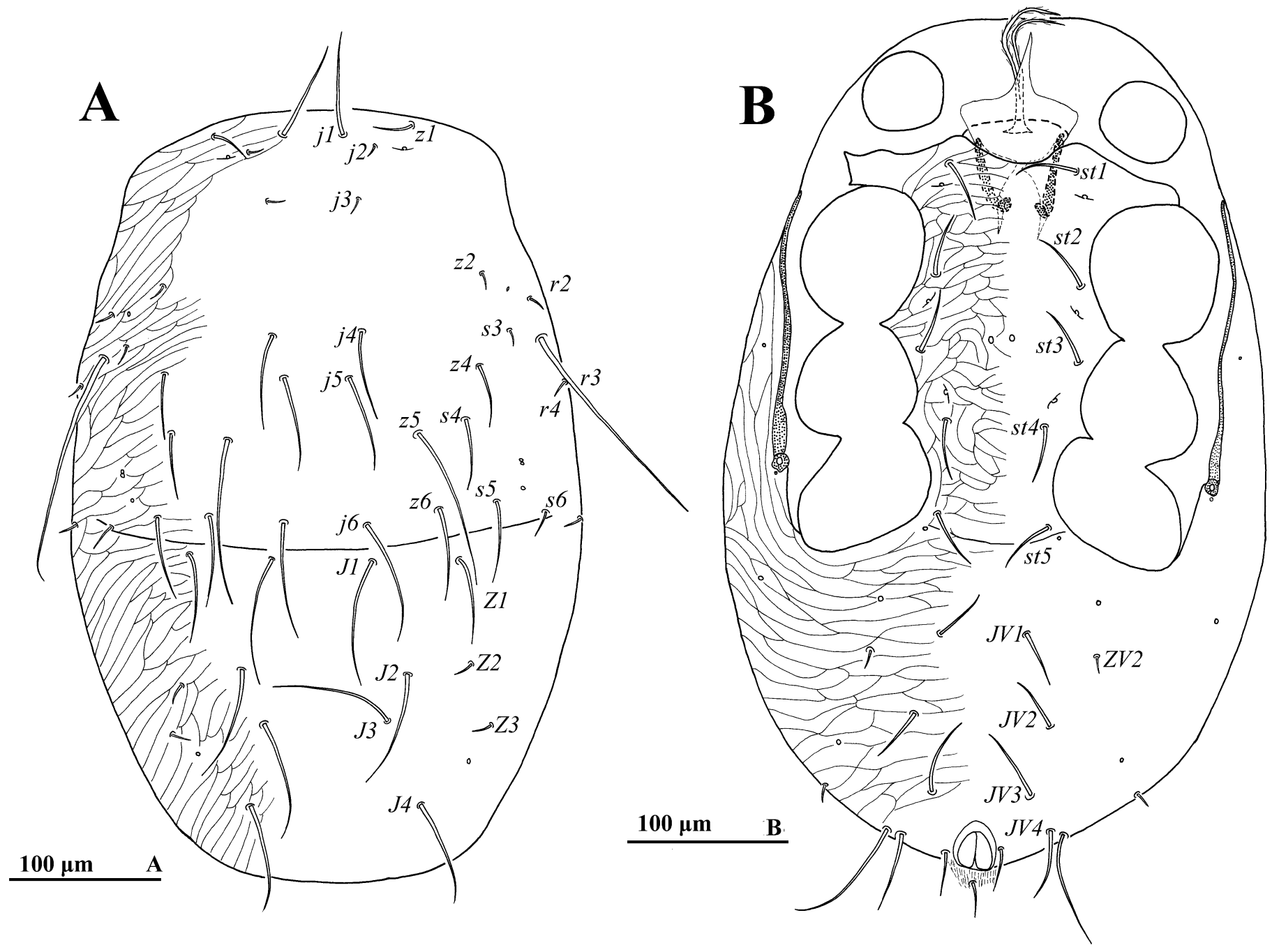

Figure 3 Psilogamasus pentatideus Yao \& Jin n. sp., male. A - Dorsum; B - Venter.

(Figure 4B) bearing four pairs of setae, setae $p c x$ (46-49) pilose, $h 1(36-38), h 2(37-40)$ and $h 3$ (48-49) smooth; deutosternal groove with 11 rows, of which anterior ten rows with numerous irregular teeth, and posterior one linear; corniculus (18-22) and internal mala acute as in female. Movable digit of chelicera with five teeth, proximal one larger, and arthrodial brush at base; fixed digit with nine teeth (Figure 4C). Palp 215-221 in length, as in female; trochanter, femur and genu as shown in Figure 4D.

Legs (Figure 4E) - Lengths of legs I-IV 879-894, 585-597, 545-559 and 922-968, respectively. Leg IV longer than other legs, Leg II stouter than others. Chaetotaxy: Setal complement and arrangement as in female. Two ventral setae on femur II modified into spurs fused at base; one ventral seta on genu II and tibia II each modified into spur. Other characteristics as in female.

\section{Other stage}

Unknown. 


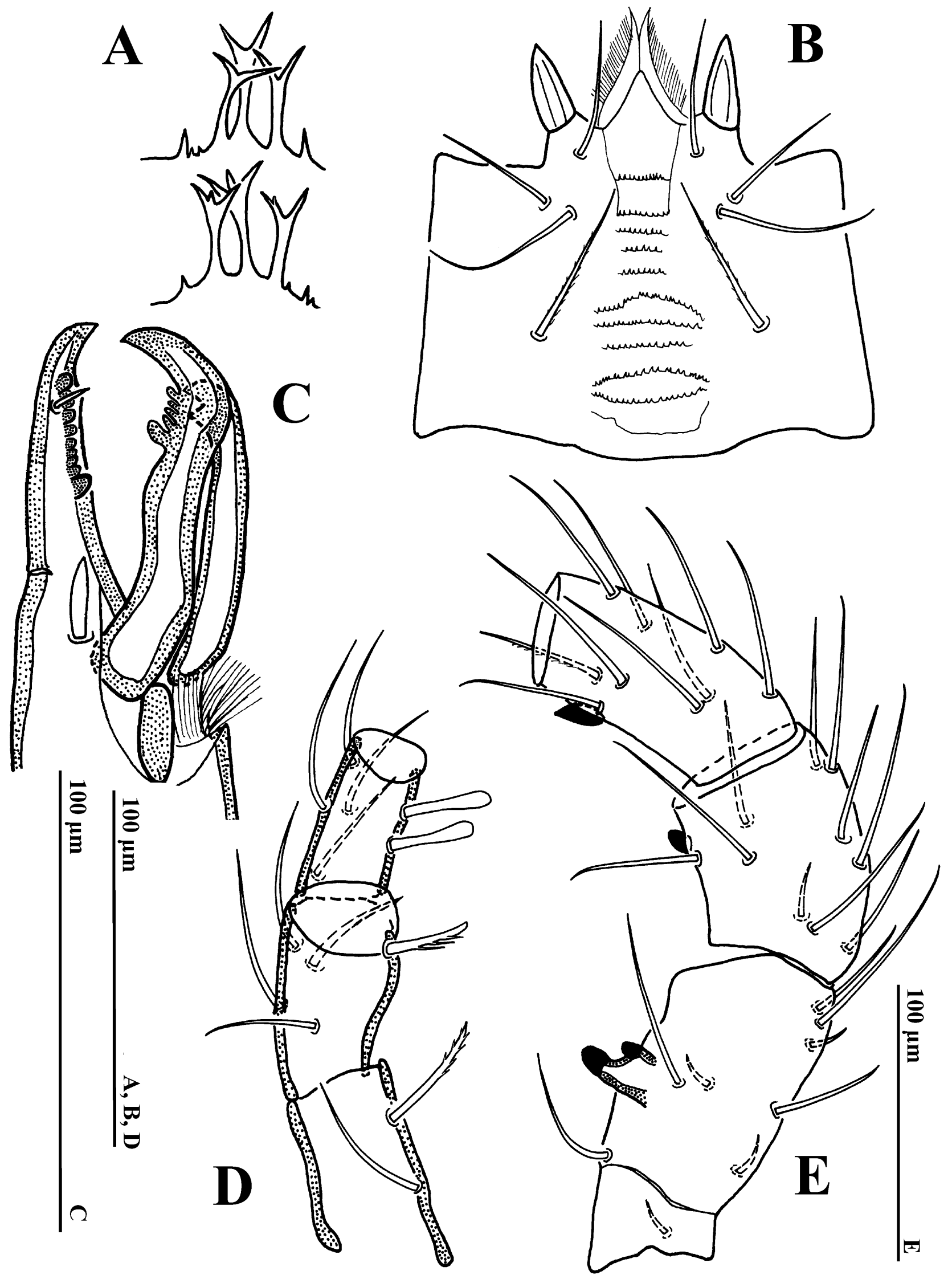

Figure 4 Psilogamasus pentatideus Yao \& Jin n. sp., male. A - Epistome; B - Subcapitulum; C - Chelicera; D - Palpus; E - Femur, genu and tibia of leg II (posterolateral view) 


\section{Etymology}

This species is named after its movable digit of chelicera in male with five teeth, what is rare in Parasitidae.

\section{Remarks}

The newly described species is morphologically similar to $P$. pentasetosus in the setal number on the opisthonotal shield and dental number of chelicera movable digit. However, P. pentatideus Yao \& Jin n. sp. is different from P. pentasetosus by follows: (1) podonotal shield bearing 18 pairs of setae, vs. 16 pairs in P. pentasetosus; (2) opisthogastric shield bearing five pairs of setae, vs. 6 pairs in the latter; (3) three prongs of epistome each apically divided into two to five branches, vs. central one multidentate distally and lateral ones spiculate or bifid apically in P. pentasetosus.

\section{Key to known species of the genus Psilogamasus}

\section{Females}

1. Opisthonotal shield with six pairs of setae; seta $Z 3$ on shield $\ldots \ldots \ldots \ldots \ldots \ldots \ldots \ldots 2$

— Opisthonotal shield with five pairs of setae, seta $Z 3$ off shield $\ldots \ldots \ldots \ldots \ldots \ldots \ldots \ldots$

2. Podonotal setae $\mathrm{j} 1$ feathered; pa and po equal in length; fixed digit of chelicera with five

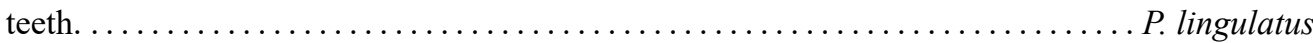
— Podonotal setae j1 smooth; pa shorter than po in length; fixed digit of chelicera with seven

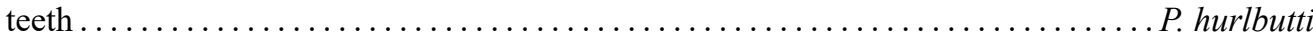

3. Peritrematal groove extending anteriorly and close to posterior end of coxa III; movable

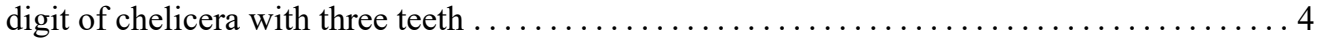
- Peritrematal groove extending anteriorly to beyond coxa II; movable digit of chelicera with four teeth .................................................... 5

4. Epigynium serrate on both sides; the base of epistome smooth P. brachysternalis — Epigynium smooth on both sides; the base of epistome with small denticles. P. longascidiformis

5. Opisthogastric shield with six pairs of setae; dorsal seta $\mathrm{r} 4$ off shield .......P. pentasetosus — Opisthogastric shield with five pairs of setae; dorsal seta $\mathrm{r} 4$ on shield .. P. pentatideus $\mathbf{n}$. sp.

\section{Males}

1. movable digit of chelicera with five teeth; three prongs of epistome each apically divided into two to five branches................................ pentatideus $\mathbf{n} . \mathbf{s p}$. - movable digit of chelicera with six teeth, central prong of epistome multidentate distally and lateral ones spiculate or bifid apically P. hurlbutti

\section{Acknowledgements}

We express our thanks to two reviewers for their insightful comments on the manuscript. We sincerely thank Dr. Owen Seeman offered the help to revision for English writing and comments. This work was supported by the National Natural Science Foundation of China (31872275), the Provincial Science Fund for Distinguished Young Scholars ([2016]5641). 


\section{References}

Athias-Henriot C. 1969. Psilogamasus hurlbutti, n. g., n. sp., gamaside nouveau de Tanzani (Acariens Anactinotriches, Parasitidae). Ann. Soc. Entomol. Fr., 5: 439-449.

Athias-Henriot, C. 1971. La divergence néotaxique des Gamasides (Arachnides). Bull. sci. Bourgogne, 28: $93-106$.

Athias-Henriot, C. 1975. Nouvelles notes sur les Amblyseiini II. Le relevé organotaxique de la face dorsaleadulte (Gamasides, Protoadenique, Phytoseiidae). Acarologia, 17: 20-29.

Bai, X.L. \& Ma, L.M. 2013. A new species of the genus Vulgarogamasus and the supplementary description of female of Neogamasus Crispus Ma \& Yan (Acari, Parasitidae). Acta Zootaxonomica Sinica, 38(1): 70-74. (In Chinese)

Evans, G.O. 1963a. Observation on the chaetotaxy of the legs in the free-living Gamasina (Acari: Mesostigmata). Bull. br. Mus. (nat. Hist.) Zool., 10: 275-303. doi:10.5962/bhl.part.20528

Evans G.O. 1963b. Some observations on the chaetotaxy of the pedipalps in the Mesostigmata (Acari). Ann. Mag. Nat. Hist. (Ser. 13), 6: 513-527. doi:10.1080/00222936308651393

Halliday, R. B., Walter, D. E. \& Polak, M. 2005. A new species of Gamasodes Oudemans from Australia (Acari: Parasitidae). Zootaxa, 1001(1): 17-30. doi:10.11646/zootaxa.1001.1.2

Hennessey M.K., Farrier M.H. 1989. Mites of the Family Parasitidae (Acari: Mesostigmata) Inhabiting Forest Soils of North and South Carolina. N.C. ARS. Tech. Bull., 291. pp. 78.

Hrúzová, K. \& Fend'a, P. 2018. The family Parasitidae (Acari: Mesostigmata)-history, current problems and challenges. Acarologia, 58(Suppl): 25-42. doi:10.24349/acarologia/20184280

Hyatt, K.H. 1980. Mites of the subfamily Parasitinae (Mesostigmata: Parasitidae) in the British Isles. Bull. Br. Mus. (Nat. Hist.), Zool., 38(5): 237-378.

Juvara-Bal, I. 2019. Occigamasus, a new genus of pergamasine mites, with description of two new species from the west coast of North America (Parasitiformes: Gamasina: Parasitidae). Acarologia, 59: 551-570. doi:10.24349/acarologia/20194354

Ma, L.M. \& Lin, J.Z. 2005. Four new species of the family Parasitidae (Acari, Gamasina). Acta Zootaxonomica Sinica, 21(2): 76-82. (In Chinese)

Micherdziński, W. 1969. Die Familie Parasitidae Oudemans, 1901 (Acarina, Mesostigmata). Krakow (Panstwowe Wydawnictwo Naukowe), 690 pp. (with Polish and Russian summaries)

Tichomirov, S.I. 1969. Morphological and ecological structure of the genus Parasitus sensu Micherdziński, 1966 (Gamasoidea, Parasitidae). I. Subgenera Eugamasus Berl., Parasitus Latr., Vulgarogamasus subgen. n.]. Zool. Zh., 48: 1325-1336.

Tseng, Y.H. 1995. A taxonomically study of free-living Gamasine mite family Parasitidae Oudemans (Acari, Mesostigmata) from Taiwan. J. Taiwan Mus., 48(2): 11-81.

Witaliński, W. 2017. A new species of Trachygamasus from Poland, a new definition of the genus, and a key to the world species (Parasitiformes: Parasitidae). Zootaxa, 4303(3): 407-416. doi: 10.11646/zootaxa.4303.3.6

Yao, M.Y., Yi, T.C., Guo, J.J., Jin, D.C., \& Zhang, R.Z. 2019. Three new species of Trachygamasus (Mesostigmata: Parasitidae) from China, with a key to world species of the genus. Syst. Appl. Acarol., 24(8): 1465-1489. doi:10.11158/saa.24.8.9

Yao, M.Y., Long Y., Yi, T.C., Guo, J.J., Jin, D.C., \& Zhang, R.Z. 2020. Description of two new species of Trachygamasus Berlese (Mesostigmata: Parasitidae) and the male of Trachygamasus multisetus from China, with notes on the genus. Syst. Appl. Acarol., 25(4): 633-648. doi:10.11158/saa.25.4.4 